\title{
SMAA-AD Model in Multicriteria Decision-Making Problems with Stochastic Values and Uncertain Weights
}

\author{
Feng Yang • Fuguo Zhao • Liang Liang • \\ Zhimin Huang
}

Received: 30 August 2012 / Revised: 25 December 2012 / Accepted: 20 April 2013 /

Published online: 3 April 2014

(C) Springer-Verlag Berlin Heidelberg 2014

\begin{abstract}
The current paper considers the stochastic multicriteria decision-making (MCDM) problems with multiple alternatives, stochastic criterion values and uncertain criterion weights. We propose SMAA-AD model and illustrate how SMAA-AD model is used in such stochastic MCDM problems. In SMAA-AD model, absolute dominant method is used to turn stochastic criterion values into deterministic absolute dominant values, and stochastic multicriteria acceptability analysis (SMAA) is used to rank the alternatives without foreknowing the decision maker' preference on criterion weights. SMAA-AD model provides three indices, i.e., rank acceptability index, holistic acceptability index and central weight vector, to support the decision in the stochastic MCDM problems. SMAA-AD model overcomes some shortcomings of traditional MCDM methods. For example, it needs not to predefine any parameters and functions. We use a case of technology competition for cleaning polluted soil in Helsinki to illustrate our method.
\end{abstract}

Keywords Multicriteria decision-making (MCDM) - Stochastic multicriteria acceptability analysis (SMAA) - Absolute dominant method (ADM) - Stochastic value . Uncertain weight

F. Yang $\cdot$ F. Zhao $\cdot$ L. Liang

School of Management, University of Science and Technology of China, 96 Jinzhai Road, Hefei 230026, Anhui, People's Republic of China

Z. Huang

Robert B. Willumstad School of Business, Adelphi University, Garden City,

Long Island, NY 11530, USA

Z. Huang $(\varangle)$

School of Management and Economics, Beijing Institute of Technology,

Beijing 100081, People's Republic of China

e-mail: huang@adelphi.edu 


\section{Introduction}

The current paper focuses on the stochastic multicriteria decision-making (MCDM) problems with more than one alternatives where the decision maker (DMer) is requested to rank the alternatives according to multiple criteria. Different from the traditional MCDM problems, in the stochastic MCDM problems, the criterion values (i.e., the values of different criteria for any given alternative) is stochastic, and the criterion weights (i.e., the weights or importance degrees of different criteria) is uncertain. The forthcoming two paragraphs show that stochastic criterion values and uncertain criterion weights are often observed in many real-world MCDM problems.

When dealing with many MCDM problems, we often feel it difficult to provide deterministic measurements for the criterion values. For example, in some cases with limitation of time and capability, the DMer cannot provide deterministic criterion values. Hence the use of accurate criterion values in such a decision making procedure means an oversimplification of the problem. If multiple DMers are involved in such a procedure, it turns more difficult to reach consensus about exact measurement of each criterion. As a result, the criterion measurements are usually obtained in the form of stochastic values or multiple deterministic values with the corresponding probabilities. For the case of stochastic values, the DMer just needs to specify the upper and lower bounds of each criterion value. Considering the total lack of knowledge about the probability distributions of the criteria measurements, we assume that the criteria measurements distribute uniformly within the specific bound.

Another aspect of the actual MCDM problems is related to the difficulty of obtaining the preference information of different DMers about the criterion weights. Sometimes the DMers are not willing to express their preferences explicitly. Sometimes the DMers are unable to estimate the importance degrees of different criteria especially when some criteria are monetary while the others are nonmonetary. In addition, the relative importance of different criteria will not keep steady with changing contexts. As a result, it is difficult for the DMer to gain the explicit importance weight about the criteria. Thus only inaccuracy or incomplete weights are available. Lahdelma and Salminen [9] proposed five types of restrictions on the weight space: (1) partial or complete ranking of criteria, (2) intervals for weights, (3) intervals for weight ratios, (4) linear inequality constraints for weights, and (5) nonlinear inequality constraints for weights.

Several methods have been proposed for dealing with MCDM problems with uncertain criterion weights. Some methods are based on multiattribute utility theory [7] where the uncertainty of the criterion weights is represented by probability distributions and the alternatives are evaluated according to their expected utility. In ELECTRE methods [15], the uncertainty is handled by the threshold model. In PROMETHEE methods [13], flow calculations with intervals are performed as extensions of the current calculations. The PRIME method [16] represents the uncertainty by intervals for weight ratios. In addition, [2] introduced Fuzzy MCDM method to represent the uncertainty by fuzzy numbers.

Stochastic multicriteria acceptability analysis (SMAA) is a family of methods that tackle various classifications of MCDM problems with uncertain, imprecise, or missing information about criterion weights. A lot of extended SMAA methods have be pro- 
posed in choosing, ranking, or sorting candidates in the MCDM problems [5]. SMAA is based on the overall compromise criterion method and comparative hypervolume criterion. Bana e Costa [1] introduced the overall compromise criterion method to calculate the amount of difference between the preferences of various DMers by defining a joint probability density function for the weight space. Comparative hypervolume criterion by Charnetski [3] is based on computing the volume of the multidimensional weight space that makes each alternative the most preferred one. In the original SMAA method, inverse weight space analysis is introduced to identify for each alternative the weights that make it most preferred based on an additive utility or value function. SMAA-2 extends the analysis to a general utility or value function. SMAA-2 method also provides some useful tools for ranking the alternatives, and those tools include rank acceptability indices, central weight vectors, confidence factors and others. Lahdelma and Salminen [10] developed cross confidence factors which were based on computing confidence factors for alternatives by using each other's central weight vectors when the problem involved a large set of efficient alternatives. Lahdelma et al. [8] introduced a method to handle dependent uncertainties in stochastic multicriteria group decision-making problems based on SMAA-2. SMAA-O method [14] extends SMAA-2 for treating mixed ordinal and cardinal criteria. SMAA-3 [17] uses the outranking pseudo-criteria, not a utility function, in ELECTRE III procedure. SMAA-D [11] is a variant of SMAA-2 that employs the efficiency scores of data envelopment analysis instead of a value/utility function. SMAA-P [12] assumes piecewise linear difference functions based on prospect theory instead of a utility or value function model. Ref-SMAA (SMAA-A) method [4] allows multiple DMers to use reference points and Wierzbicki's achievement scalarizing function to provide descriptive information for each alternative.

In the original SMAA, a utility or value function models the DMer's preference structure. However, the utility or value function is not linear because of diminishing marginal utility when dealing with the real-life problems. It is difficult to define the exact function. In SMAA-3, ELECTRE III-type pseudo-criteria are used instead. The DMer needs to provide deterministic thresholds and cutting levels. The results will vary greatly if the DMer chooses different parameters. SMAA-P assumes linear prospect theory type difference functions and avoids the drawback of linear utility or value function model, while the DMer needs to choose reference points and coefficients of loss aversion in advance. It is difficult to predefine the parameters, and the results depend on the assumed parameters. In summary, all of the above methods need to foreknow the DMer's preference on utility function, value function, threshold, parameters and so on.

In this paper, we introduce a new method to transform stochastic criteria measurements to deterministic ones without needing the foreknowledge of the DMer's preference. The basis of the approach is to calculate how much one alternative is better than all other alternatives absolutely by accumulated pairwise integrating without presetting any parameters. When we obtain the deterministic absolute dominant values, the inverse weight space analysis is used to describe each alternative what kind of preferences makes it the most preferred one, or places it on any particular rank. We will use three indices to support the decision. The first and second are the acceptability indices which are to measure the variety of different preferences that support an alter- 
Table 1 A typical multicriteria decision matrix with bounds of stochastic criterion values

\begin{tabular}{ccccc}
\hline & Criterion 1 & Criterion 2 & $\ldots$ & Criterion $m$ \\
\hline Alternative 1 & {$\left[x_{11}^{l}, x_{11}^{u}\right]$} & {$\left[x_{12}^{l}, x_{12}^{u}\right]$} & $\ldots$ & {$\left[x_{1 m}^{l}, x_{1 m}^{u}\right]$} \\
Alternative 2 & {$\left[x_{21}^{l}, x_{21}^{u}\right]$} & {$\left[x_{22}^{l}, x_{22}^{u}\right]$} & $\ldots$ & {$\left[x_{2 m}^{l}, x_{2 m}^{u}\right]$} \\
$\vdots$ & $\vdots$ & $\vdots$ & $\vdots$ & $\vdots$ \\
Alternative $n$ & {$\left[x_{n 1}^{l}, x_{n 1}^{u}\right]$} & {$\left[x_{n 2}^{l}, x_{n 2}^{u}\right]$} & $\ldots$ & {$\left[x_{n m}^{l}, x_{n m}^{u}\right]$} \\
\hline
\end{tabular}

native, and the third is the central weight vectors representing the typical preferences which make an alternative preferred.

This paper is organized as follows. In Sect. 2, we introduce absolute dominant method (ADM) which transforms stochastic criteria measurements to deterministic ones. In Sect. 3, we propose SMAA-AD method that combines the fundamental principle of SMAA-2 with ADM to obtain rank acceptability index and central weight vector of each alternative. In Sect. 4, we demonstrate the use of the SMAA-AD method in a technology competition for cleaning polluted soil in Helsinki. We end this paper with conclusions in the last section.

\section{Absolute dominant method (ADM)}

We consider stochastic MCDM problems, where the DMer wants to identify one or a few 'best' alternatives from a set of $n$ alternatives $\left\{x_{1}, x_{2}, \cdots, x_{n}\right\}$. The alternatives are evaluated in terms of $m$ criteria. The criterion values are represented by stochastic variables $x_{i k}$ with estimated probability distributions and density functions $f\left(x_{i k}\right)$ in the criterion space $X \subseteq R^{n \times m}$. The upper bound of $x_{i k}$ is $x_{i k}^{u}$ and the lower bound is $x_{i k}^{l}$. The stochastic MCDM problem is described as Table 1 .

We define absolute dominant function $V(\cdot)$. It represents the expectation that how much alternative $i$ is better than alternative $j$. For the criterion to be maximized, the absolute dominant value that alternative $i$ is better than alternative $j$ on criterion $k$ is

$$
V_{i j k}=\iint_{D}\left(x_{i k}-x_{j k}\right) f\left(x_{i k}, x_{j k}\right) d x_{i k} d x_{j k}
$$

Here $D=\left\{\left(x_{i k}, x_{j k}\right) \mid x_{i k}^{l} \leq x_{i k} \leq x_{i k}^{u}, x_{j k}^{l} \leq x_{j k} \leq x_{j k}^{u}, x_{i k}>x_{j k}\right\}$.

While for the criterion to be minimized

$$
V_{i j k}=\iint_{D^{\prime}}\left(x_{j k}-x_{i k}\right) f\left(x_{i k}, x_{j k}\right) d x_{i k} d x_{j k}
$$

where $D^{\prime}=\left\{\left(x_{i k}, x_{j k}\right) \mid x_{i k}^{l} \leq x_{i k} \leq x_{i k}^{u}, x_{j k}^{l} \leq x_{j k} \leq x_{j k}^{u}, x_{j k}>x_{i k}\right\}$.

Here $f\left(x_{i k}, x_{j k}\right)$ is the joint density function of $x_{i k}$ and $x_{j k}$. Usually $x_{i k}$ and $x_{j k}$ are independent stochastic variables, thus $f\left(x_{i k}, x_{j k}\right)=f\left(x_{i k}\right) f\left(x_{j k}\right)$. It is assumed without loss of generality that every criterion is to be maximized and the alternatives 
Fig. 1 The integral area if the values of $x_{i k}$ and $x_{j k}$ have a cross area

Fig. 2 The integral area if $x_{i k}^{l}>x_{j k}^{u}$
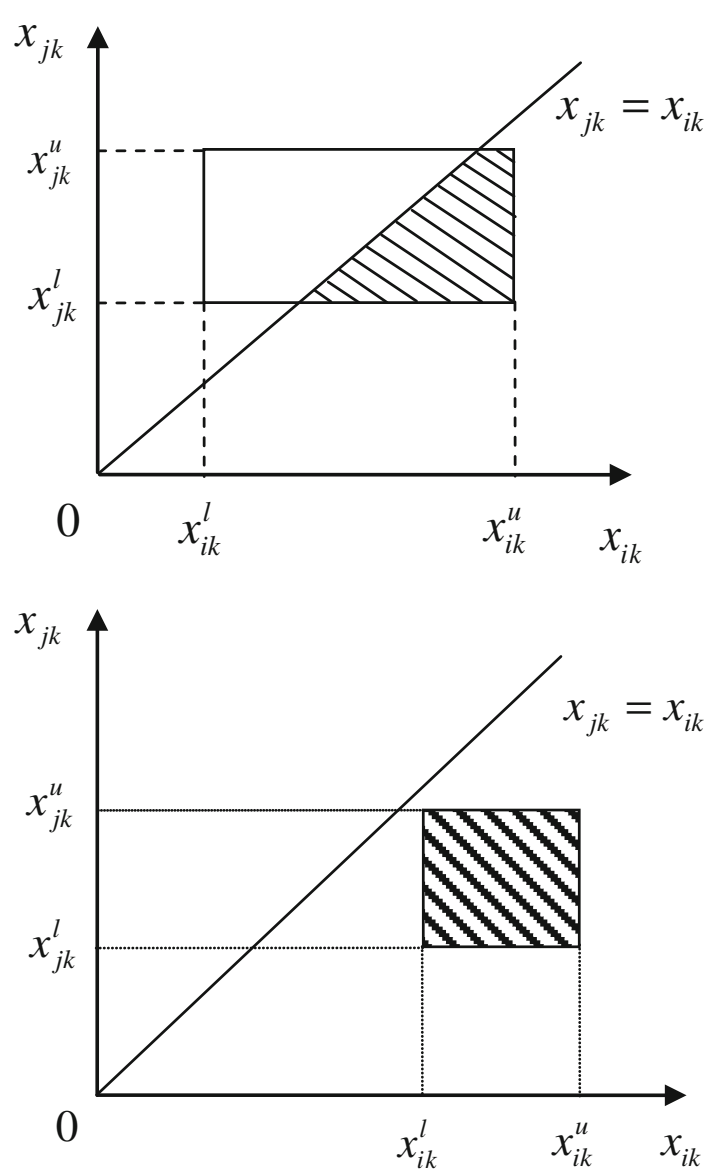

are independent. The following four cases describe the calculation of the absolute dominant value.

Case 1. Both $x_{i k}$ and $x_{j k}$ are stochastic

If the stochastic values of $x_{i k}$ and $x_{j k}$ have a cross area, the integral area can be illustrated graphically as in Fig. 1. If $x_{i k}^{l}>x_{j k}^{u}$, the integral area can be illustrated as in Fig. 2. Otherwise if $x_{i k}^{u}<x_{j k}^{l}$, the integral area is null-space, and the absolute dominant value is zero.

Case 2. $x_{i k}$ is stochastic and $x_{j k}$ is deterministic

If $x_{i k}$ is stochastic and $x_{j k}$ is deterministic, the joint destiny function is

$$
f\left(x_{i k}, x_{j k}\right)=f\left(x_{i k}\right) \sigma\left(x_{j k}-x_{j k}^{*}\right)
$$

Here deterministic criterion value $x_{j k}^{*}$ is a special case of independent stochastic value $x_{j k}$ with density function

$$
f\left(x_{j k}\right)=\sigma\left(x_{j k}-x_{j k}^{*}\right)
$$


Table 2 The overall absolute dominant values on each criterion

\begin{tabular}{ccccc}
\hline & Criterion 1 & Criterion 2 & $\ldots$ & Criterion $m$ \\
\hline Alternative 1 & $V_{11}$ & $V_{12}$ & $\ldots$ & $V_{1 m}$ \\
Alternative 2 & $V_{21}$ & $V_{22}$ & $\ldots$ & $V_{2 m}$ \\
$\vdots$ & $\vdots$ & $\vdots$ & $\vdots$ & $\vdots$ \\
Alternative $n$ & $V_{n 1}$ & $V_{n 2}$ & $\ldots$ & $V_{n m}$ \\
\hline
\end{tabular}

where $\sigma$ is Dirac's delta function and $f\left(x_{j k}\right)$ is the impulse function.

Then the absolute dominant function can be simplified as

$$
V_{i j k}=\int_{x_{i k}>x_{j k}^{*}}\left(x_{i k}-x_{j k}^{*}\right) f\left(x_{i k}\right) d x_{i k}
$$

Case 3. $x_{i k}$ is deterministic and $x_{j k}$ is stochastic

If $x_{i k}$ is deterministic and $x_{j k}$ is stochastic, we have

$$
V_{i j k}=\int_{x_{i k}^{*}>x_{j k}}\left(x_{i k}^{*}-x_{j k}\right) f\left(x_{j k}\right) d x_{j k}
$$

Case 4. Both $x_{i k}$ and $x_{j k}$ are deterministic

If both $x_{i k}$ and $x_{j k}$ are deterministic, the joint destiny function is defined as

$$
f\left(x_{i k}, x_{j k}\right)=\sigma\left(x_{i k}-x_{i k}^{*}\right) \sigma\left(x_{j k}-x_{j k}^{*}\right)
$$

Then absolute dominant function is simplified as

$$
V_{i j k}=x_{i k}^{*}-x_{j k}^{*}
$$

According to the above equations, we can calculate the absolute dominant value that that alternative $i$ is better than another alternative $j$. Next we consider the absolute dominant value of one alternative relative to all of other alternatives. The overall absolute dominant value that alternative $i$ is better than all of the other alternatives on criterion $k$ is

$$
V_{i k}=\sum_{j \neq i} \iint_{D}\left(x_{i k}-x_{j k}\right) f\left(x_{i k}, x_{j k}\right) d x_{i k} d x_{j k}
$$

The larger $V_{i k}$, the better alternative $i$ than all of other alternatives on criterion $k$. The overall absolute dominant values of each alternative are expressed as Table 2.

The absolute dominant value on each criterion can be normalized as

$$
v_{i k}=\frac{V_{i k}}{\max _{i} V_{i k}}
$$

Table 3 presents the normalized overall absolute dominant values on each criterion. 
Table 3 The normalized overall absolute dominant values

\begin{tabular}{lllll}
\hline & Criterion 1 & Criterion 2 & $\ldots$ & Criterion $m$ \\
\hline Alternative 1 & $v_{11}$ & $v_{12}$ & $\ldots$ & $v_{1 m}$ \\
Alternative 2 & $v_{21}$ & $v_{22}$ & $\ldots$ & $v_{2 m}$ \\
$\vdots$ & $\vdots$ & $\vdots$ & $\vdots$ & $\vdots$ \\
Alternative $n$ & $v_{n 1}$ & $v_{n 2}$ & $\ldots$ & $v_{n m}$ \\
\hline
\end{tabular}

After we get the normalized overall absolute dominant values on each criterion, the stochastic MCDM problem is converted to a deterministic MCDM problem. Then the overall absolute dominant value of alternative $i$ on all the criteria is expressed as

$$
v_{i}=\sum_{k=1}^{m} w_{k} v_{i k}
$$

where $w_{k}$ is the weight of criterion $k$. The larger $v_{i}$, the better alternative $i$ will be better than all the other alternatives. Thus the DMer will choose the alternative with the largest $v_{i}$. However, in the real world decision problems, the DMer can not provide exact weight of each criterion $w_{k}$ due to some reasons. Consequently, we propose a method that combined ADM and inverse weight space method to deal with such problems in the next section.

\section{SMAA-AD method}

The current section continues the stochastic MCDM problem in Sect. 2. According (1)(10), we have obtained the overall absolute dominant values on each criterion for any alternative. However, the DMer's preference structure on the criteria is still unknown, which makes the calculation of (11) difficult. The current section extends SMAA-2 model to SMAA-AD model by introducing ADM as discussed in the previous section. SMAA-2 uses inverse weight space analysis to describe for each alternative what kind of preferences makes it the most preferred one, or places it on any particular rank.

We assume that the DMer's preference is unknown or partially known, represented by a weight distribution with density function $f(w)$ in the set of feasible weights $W$. Here the weight space $W$ is defined as

$$
W=\left\{w \in R^{m}: w \geq 0 \text { and } \sum_{k=1}^{m} w_{k}=1\right\}
$$

The weight space is an $(n-1)$-dimensional, and can be added some constraints to represent different kinds of preference information according to different circumstances. For example, the DMer can specify precise weights $\left(w_{k}\right)$, a priority order for the criteria $\left(w_{j}>w_{k}\right.$, for some $\left.j, k\right)$, weight intervals $\left(w_{k}^{\min } \leq w_{k} \leq w_{k}^{\max }\right)$, intervals for weight ratios $\left(w_{j k}^{\min } \leq w_{j} / w_{k} \leq w_{j k}^{\max }\right)$, and arbitrary linear or non-linear constraints 
for weights $(A w \leq c$ or $g(w) \leq 0)$. Total lack of preference information is represented by a uniform weight distribution in $W$. The density function of uniform distribution is expressed as

$$
f(w)=1 / \operatorname{vol}(W)
$$

According to ADM, we define the rank of each alternative as an integer from the best rank $(=1)$ to the worst rank $(=n)$ by means of a ranking function

$$
\operatorname{rank}(i, w)=1+\sum_{j \neq i} \rho\left(v_{j}>v_{i}\right)
$$

where $\rho($ true $)=1$ and $\rho($ false $)=0$. The favorable weights sets for each alternative to get rank $r$ is defined by

$$
W_{i}^{r}=\{w \in W: \operatorname{rank}(i, w)=r, \quad r=1,2, \cdots, n\}
$$

Any weight $w \in W_{i}^{r}$ makes alternative $i$ obtain rank $r$.

Next we use three descriptive indices to appraise all of the alternatives in the stochastic MCDM problem.

(1) Rank Acceptability Index $b_{i}^{r}$

The first descriptive measure of SMAA-AD model is rank acceptability index $b_{i}^{r}$, which measures the variety of different preferences that grant alternative $x_{i}$ to get rank $r . b_{i}^{r}$ is computed numerically as a multidimensional integral over the favorable rank weights by

$$
b_{i}^{r}=\int_{W_{i}^{r}} f(w) d w
$$

In fact, the rank acceptability index can be regarded as the probability for which alternative $x_{i}$ gets rank $r$ if the weights distribute uniformly in the feasible weight space. Evidently, the rank acceptability indices are in the range $[0,1]$ where 0 indicates that the alternative will never obtain a given rank and 1 indicates that it will obtain the given rank always with any choice of weights.

Among all of $b_{i}^{r}$, we focus most attention on the first rank acceptability index $b_{i}^{1}$. The first rank acceptability index represents the share of all the weights space for which the alternative $i$ is the best choice. If $b_{i}^{1}$ is near to 1 , we conclude that the alternative will be selected as the best one with very high probability. If $b_{i}^{1}$ is zero or near-zero, we conclude that the alternative is hardly to be regarded the best one. In summary, the most acceptable alternatives are those with high values of $b_{i}^{1}$.

\section{(2) Holistic Acceptability Index $\boldsymbol{a}_{\boldsymbol{i}}^{\boldsymbol{h}}$}

The holistic acceptability index $a_{i}^{h}$ aims at measuring the overall acceptability of each alternative, and it is computed as

$$
a_{i}^{h}=\sum_{r=1}^{n} \alpha^{r} b_{i}^{r}
$$


Here $\alpha_{r}$ is the so-called meta-weight, and $1 \geq \alpha_{1} \geq \alpha_{2} \geq \cdots \geq \alpha_{n} \geq 0$. How to determine the meta-weights has been discussed in [9]. In general, the metaweights are normalized, nonnegative and nonincreasing when the rank increases. Usually we set the forms of $\alpha_{r}$ as linear weights $\alpha^{r}=(n-r) /(n-1)$, inverse weights $\alpha^{r}=1 / i$, or centroid weights $\alpha^{r}=\sum_{i=r}^{n} 1 / i / \sum_{i=r}^{n} 1 / i$.

(3) Central Weight Vector $w_{i}^{c}$

Central weight vector $w_{i}^{c}$ is regarded as the expected centroid of the favorable weight space. It is the most likely weight vector that represents a typical DM prefers an alternative. It is computed as an integral of the weight vector over the weight distributions by

$$
\begin{array}{r}
w_{i}^{c}=\int_{W_{i}^{1}} w f(w) d w / \int_{W_{i}^{1}} f(w) d w \\
=\int_{W_{i}^{1}} w f(w) d w / b_{i}^{1}
\end{array}
$$

\section{Illustration}

In the current section we demonstrate the use of SMAA-AD method in the context of multicriteria decision support in a technology competition for cleaning polluted soil in Helsinki [6]. The city of Helsinki in Finland was planning to clean ex-industrial contaminated areas. There were nine candidates to compete for the business. The DMers were eight experts from different fields, and they were responsible for evaluating the candidates. In the first step, the DMers would choose three best candidates to testclean a small part of the contaminated region. Then the winner of the test-cleaning phase will get the contract for cleaning the whole area. As a result, the problem was converted to how the DMers would choose the three best candidates. To deal with this problem, the DMers chose five criteria to evaluate the nine alternatives: (1) costs, (2) environment, (3) innovation, (4) credibility and (5) project management (Table 4).

Only the costs criterion could be quantified directly, thus the DMers had to define scales for the other criteria and assess criterion values for each alternative to compare among the alternatives. The following scale of criteria was agreed on after discussion. Only the costs criterion was to be minimized, the other four criteria were all to be maximized. The cost criterion measurements were provided by the candidates in the contract offers and were measured in million FIM. Hokkanen [6] described the criteria scales as follows:

Table 5 presents the criterion values for each alternative that the DMers have reached consensus. All the criterion values are the average evaluations of the DMers. Each criteria measurement is given as an interval $x_{i k} \pm \Delta x_{i k}$ to represent the imprecision or uncertainty of each criterion. The bottom row of Table 5 shows the intervals that the DMers defined for each criterion. The uncertainty of the cost criterion is assumed to be $\pm 10 \%$ for all alternatives except $\mathrm{X}_{1}$ and $\mathrm{X}_{6}$ for which much larger intervals are used. 
Table 4 The scales of four criteria

\begin{tabular}{|c|c|}
\hline \multicolumn{2}{|c|}{ Environment } \\
\hline 1 & $\begin{array}{l}\text { Environmental effects were controlled by isolation, i.e. the chemicals were } \\
\text { trapped or encapsulated between tight layers of soil and/or concrete } \\
\text { materials. }\end{array}$ \\
\hline 2 & $\begin{array}{l}\text { Environmental effects were controlled by strobilization, i.e. binding the } \\
\text { harmful chemicals to an insoluble form. }\end{array}$ \\
\hline 3 & $\begin{array}{l}\text { Environmental effects were controlled by degradation, i.e. by composting } \\
\text { or burning the material. }\end{array}$ \\
\hline \multicolumn{2}{|c|}{ Innovation } \\
\hline 1 & The method had been already commercially used in Finland. \\
\hline 2 & The method was new in Finland but in commercial use somewhere. \\
\hline 3 & The method was based on a new idea in this context and was new in Finland. \\
\hline 4 & The method was universally new. \\
\hline \multicolumn{2}{|c|}{ Credibility } \\
\hline 1 & It was doubtful that the suggested method worked in this task. \\
\hline 2 & It was possible that the suggested method fitted this problem. \\
\hline 3 & It was no doubt that the suggested method worked in practice. \\
\hline \multicolumn{2}{|c|}{ Project management } \\
\hline 1 & These factors* were not described in the proposal. \\
\hline 2 & These factors* were partly taken into account in the proposal. \\
\hline 3 & These factors* were all taken into account in the proposal. \\
\hline
\end{tabular}

* Quality control, control of permission procedure, and cooperation with the authorities

Table 5 The criterion values and associated uncertainty intervals

\begin{tabular}{llllll}
\hline Alternative & $\begin{array}{l}\text { Criterion 1 } \\
\text { cost }\end{array}$ & $\begin{array}{l}\text { Criterion 2 } \\
\text { environment }\end{array}$ & $\begin{array}{l}\text { Criterion 3 } \\
\text { innovation }\end{array}$ & $\begin{array}{l}\text { Criterion 4 } \\
\text { credibility }\end{array}$ & $\begin{array}{l}\text { Criterion 5 } \\
\text { proj. mngt }\end{array}$ \\
\hline $\mathrm{X}_{1}$ & $19 \pm 5.9^{*}$ & 2 & 3 & 1 & 1 \\
$\mathrm{X}_{2}$ & 2.3 & 1 & 1.8 & 2 & 2 \\
$\mathrm{X}_{3}$ & 6 & 2 & 1.9 & 2 & 1.1 \\
$\mathrm{X}_{4}$ & 3.6 & 2.3 & 2 & 1.5 & 1.2 \\
$\mathrm{X}_{5}$ & 8.4 & 2.2 & 2.7 & 2 & 2 \\
$\mathrm{X}_{6}$ & $13.6 \pm 2.85^{*}$ & 2.8 & 2.8 & 2 & 2 \\
$\mathrm{X}_{7}$ & 5.2 & 1 & 1 & 2.8 & 3 \\
$\mathrm{X}_{8}$ & 5.6 & 2 & 2 & 2.7 & 2.9 \\
$\mathrm{X}_{9}$ & 6 & 2 & 1 & 2.5 & 3 \\
$\Delta x$ & $\pm 10 \%$ & \pm 0.5 & \pm 0.3 & \pm 0.3 & \pm 0.3 \\
\hline
\end{tabular}

* Specified intervals apply for costs of $\mathrm{X}_{1}$ and $\mathrm{X}_{6}$ instead of $\pm 10 \%$

The weights of different criteria are presented in Table 6. Since the DMers do not reach an agreement on the importance of each criterion, the weight intervals are given in the form of DMers' minimum and maximum weights. In our analysis, the criteria and weights all follow uniform distributions in the corresponding intervals.

Table 7 presents the results based on 500,000 simulations of the normalized overall absolute dominant values and weight vectors. The aim is to choose three best 
Table 6 DMers' minimum and maximum weights

\begin{tabular}{llllll}
\hline Weights & $\begin{array}{l}\text { Criterion 1 } \\
\text { costs }\end{array}$ & $\begin{array}{l}\text { Criterion 2 } \\
\text { environment }\end{array}$ & $\begin{array}{l}\text { Criterion 3 } \\
\text { innovation }\end{array}$ & $\begin{array}{l}\text { Criterion 4 } \\
\text { credibility }\end{array}$ & $\begin{array}{l}\text { Criterion 5 } \\
\text { project management }\end{array}$ \\
\hline $\min$ & 0.08 & 0.19 & 0.20 & 0.06 & 0.06 \\
$\max$ & 0.38 & 0.33 & 0.42 & 0.22 & 0.15 \\
\hline
\end{tabular}

Table 7 The holistic acceptability index $a^{h}$ and rank acceptability index $b^{r}(\%)$

\begin{tabular}{lllllllllll}
\hline Alternative & $a^{h}$ & $b^{1}$ & $b^{2}$ & $b^{3}$ & $b^{4}$ & $b^{5}$ & $b^{6}$ & $b^{7}$ & $b^{8}$ & $b^{9}$ \\
\hline $\mathrm{X}_{1}$ & 32.03 & 0 & 0 & 5.95 & 23.39 & 9.52 & 9.34 & 11.03 & 15.42 & 25.35 \\
$\mathrm{X}_{2}$ & 25.36 & 0.06 & 0.92 & 1.23 & 6.58 & 11.18 & 12.21 & 24.59 & 25.60 & 17.68 \\
$\mathrm{X}_{3}$ & 6.85 & 0 & 0 & 0 & 0 & 0 & 0.18 & 4.31 & 45.68 & 49.83 \\
$\mathrm{X}_{4}$ & 53.93 & 0.50 & 5.00 & 16.27 & 22.20 & 26.55 & 18.62 & 10.85 & 0 & 0 \\
$\mathrm{X}_{5}$ & 69.15 & 0 & 21.68 & 44.56 & 12.46 & 12.98 & 3.24 & 5.09 & 0 & 0 \\
$\mathrm{X}_{6}$ & 97.34 & 83.71 & 13.39 & 1.20 & 1.39 & 0.18 & 0.12 & 0 & 0 & 0 \\
$\mathrm{X}_{7}$ & 34.63 & 0 & 1.03 & 6.70 & 8.73 & 13.12 & 19.13 & 31.98 & 12.17 & 7.14 \\
$\mathrm{X}_{8}$ & 85.10 & 15.78 & 57.98 & 17.93 & 7.87 & 0.45 & 0 & 0 & 0 & 0 \\
$\mathrm{X}_{9}$ & 45.61 & 0 & 0 & 6.16 & 17.38 & 26.03 & 37.16 & 12.15 & 1.13 & 0 \\
\hline
\end{tabular}

alternatives, thus the metaweights of top three ranks should not be different greatly. Therefore, we choose the linear weights $\alpha^{r}=(n-r) /(n-1)$ to calculate the holistic acceptability index.

Based on the first rank acceptability indices, the top three alternatives are $\mathrm{X}_{6}, \mathrm{X}_{8}$ and $\mathrm{X}_{4} . \mathrm{X}_{6}$ has the highest acceptability index $(83.71 \%)$ for the best rank, and almost zero acceptability for ranks from five to nine. Alternatives $\mathrm{X}_{8}$ receive reasonably high acceptability for the top three ranks, while $\mathrm{X}_{4}$ is not. Even though the first rank acceptability index of $X_{5}$ is zero, the second and third acceptability indices are strikingly high. Therefore, we also need to consider the holistic acceptability index of each alternative.

The first three alternatives $\left(\mathrm{X}_{6}, \mathrm{X}_{8}\right.$ and $\left.\mathrm{X}_{5}\right)$ obtain markedly higher holistic acceptability indices $(97.34,85.10$ and $69.15 \%)$ and also higher rank acceptability indices for the first three ranks than the remaining alternatives. As a result, alternative $\mathrm{X}_{6}, \mathrm{X}_{8}$ and $\mathrm{X}_{5}$ are definitely the three finalists.

The central weights for each alternative are presented in Table 8. For $\mathrm{X}_{1}, \mathrm{X}_{3}, \mathrm{X}_{5}, \mathrm{X}_{7}$ and $X_{9}$, the first rank acceptability indices are all zero, which means they can not be ranked first. Therefore their central weights do not exist. The first rank acceptability index of $X_{2}$ is approximately zero; however, $X_{2}$ still has the opportunity to rank first when the number of simulations is large enough. The central weight is a reference vector that the DMers prefer to if they choose the alternative as the best one.

\section{Conclusions}

The main contribution of this paper is to propose ADM that compared the criteria measurements of each alternative and obtain the absolute dominant values. Then we 
Table 8 The central weights for the alternatives

\begin{tabular}{lllllll}
\hline Alternative & $b^{1}$ & $w_{1}$ & $w_{2}$ & $w_{3}$ & $w_{4}$ & $w_{5}$ \\
\hline $\mathrm{X}_{1}$ & 0 & - & - & - & - & - \\
$\mathrm{X}_{2}$ & 0.06 & 0.38 & 0.19 & 0.26 & 0.06 & 0.11 \\
$\mathrm{X}_{3}$ & 0 & - & - & - & - & - \\
$\mathrm{X}_{4}$ & 0.50 & 0.38 & 0.24 & 0.22 & 0.08 & 0.08 \\
$\mathrm{X}_{5}$ & 0 & - & - & - & - & - \\
$\mathrm{X}_{6}$ & 83.71 & 0.19 & 0.26 & 0.31 & 0.13 & 0.11 \\
$\mathrm{X}_{7}$ & 0 & - & - & - & - & - \\
$\mathrm{X}_{8}$ & 15.78 & 0.27 & 0.22 & 0.23 & 0.16 & 0.12 \\
$\mathrm{X}_{9}$ & 0 & - & - & - & - & - \\
\hline
\end{tabular}

combine ADM with SMAA-2 and use the deterministic values together with inverse weight space analysis to calculate the probability that each alternative could be acceptable for a particular rank.

We present a revisit of a case study showing the application of the SMAA-AD method. In the case study, uniform distributions are used to represent absence of information both in criteria measurements and preferences. The results of our reanalysis demonstrate the effectiveness of SMAA-AD model.

$\mathrm{ADM}$ has many advantages. Especially when the criterion weights are given, the stochastic MCDM problems are transformed into deterministic single-criterion decision problems, and the DMer can choose the best alternative directly. When dealing with the stochastic MCDM problems with multiple DMers, the criteria are defined in intervals subjected to specific distributions. If the weight of each criterion is also not deterministic, it can also be determined as intervals which contain the preferences of all DMers, or be given as partial (or complete) ranking of criteria. Compared with other SMAA-type methods, the advantage of the SMAA-AD method is that we need not to predefine utility function (i.e. liner utility function) and other parameters (i.e. thresholds, cutting levels reference points and coefficients of loss aversion as other SMAA-type methods). This will save a lot of time and cost in decision.

Our method is the first one to calculate the absolute dominant values by pairwise integration, and can obtain more persuasive result than traditional methods. The proposed method could be applied into more fields to achieve robust conclusions when dealing with stochastic MCDM problems. One of the unsolved problems in SMAA$\mathrm{AD}$ is how to solve problems which criteria measurements for some or all criteria are ordinal, which will be considered in future.

Acknowledgments The authors would like to thank Ministry of Education of China (Program for New Century Excellent Talents in University) and National Natural Science Foundation of China (Grant nos. 71271195, 71121061 and 71110107024) for their financial support.

\section{References}

1. Bana e Costa CA (1986) A multicriteria decision aid methodology to deal with conflicting situations on the weights. Eur J Oper Res 26:22-34 
2. Carlsson C, Fullér R (2002) Fuzzy reasoning in decision making and optimization. Physica Verlag, New York

3. Charnetski J (1973) The multiple attribute problem with partial information: the expected value and comparative hypervolume methods. Ph.D. Thesis, University of Texas at Austin

4. Durbach I (2006) A simulation-based test of stochastic multicriteria acceptability analysis using achievement functions. Eur J Oper Res 170(3):923-934

5. Figueira J, Greco S, Ehrgott M (2005) Multiple criteria decision analysis: state of the art surveys. Springer Science Business Media Inc, New York

6. Hokkanen J, Lahdelma R, Salminen P (2000) Multicriteria decision support in a technology competition for cleaning polluted soil in Helsinki. J Environ Manag 60:339-348

7. Keeney RL, Raiffa H (1976) Decisions with multiple objectives: preferences and value tradeoffs. Wiley, New York

8. Lahdelma R, Makkonen S, Salminen P (2006a) Multivariate Gaussian criteria in SMAA. Eur J Oper Res 170(3):957-970

9. Lahdelma R, Salminen P (2001) SMAA-2: stochastic multicriteria acceptability analysis for group decision making. Oper Res 49(3):444-454

10. Lahdelma R, Salminen P (2006) Classifying efficient alternatives in SMAA using cross confidence factors. Eur J Oper Res 170(1):228-240

11. Lahdelma R, Salminen P (2006b) Stochastic multicriteria acceptability analysis using the data envelopment model. Eur J Oper Res 170(1):241-252

12. Lahdelma R, Salminen P (2009) Prospect theory and stochastic multicriteria acceptability analysis (SMAA). Omega 37(5):961-971

13. Le Téno JF, Mareschal B (1998) An interval version of PROMETHEE for the comparison of building products' design with ill-defined data on environmental quality. Eur J Oper Res 109:522-529

14. Miettinen K, Lahdelma R, Salminen P (1999) SMAA-O: stochastic multicriteria acceptability analysis with ordinal data. Reports of the Department of Mathematical Information Technology, Scientific Computing, Series B, B5/1999, University of Jyväskylä

15. Roy B (1978) ELECTRE III: un algorithme de classements fondé sur une représentation floue des préférences en présence de critères multiples. Cahiers du CERO 20:3-24

16. Salo A, Hämäläinen RP (2001) Preference ratios in multiattribute evaluation (PRIME)- elicitation and decision procedures under incomplete information. IEEE Trans Syst Man Cybern 31:533-545

17. Tervonen T, Figueira J, Lahdelma R, Salminen P (2004) An inverse approach for ELECTRE III. Research Report 20/2004 of the Institute of Systems Engineering and Computers (INESC-Coimbra), Coimbra, Portugal

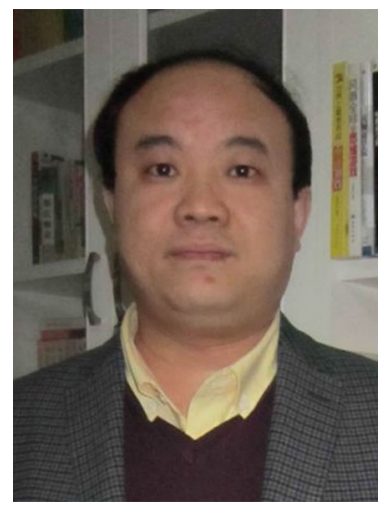

Feng Yang received the Ph.D. degree in Management Science and Engineering from University of Sciencen and Technology of China, Anhui, China in 2006. He is currently full professor at School of Management, University of Science and Technology of China. His research interests include decision science and supply chain management. 

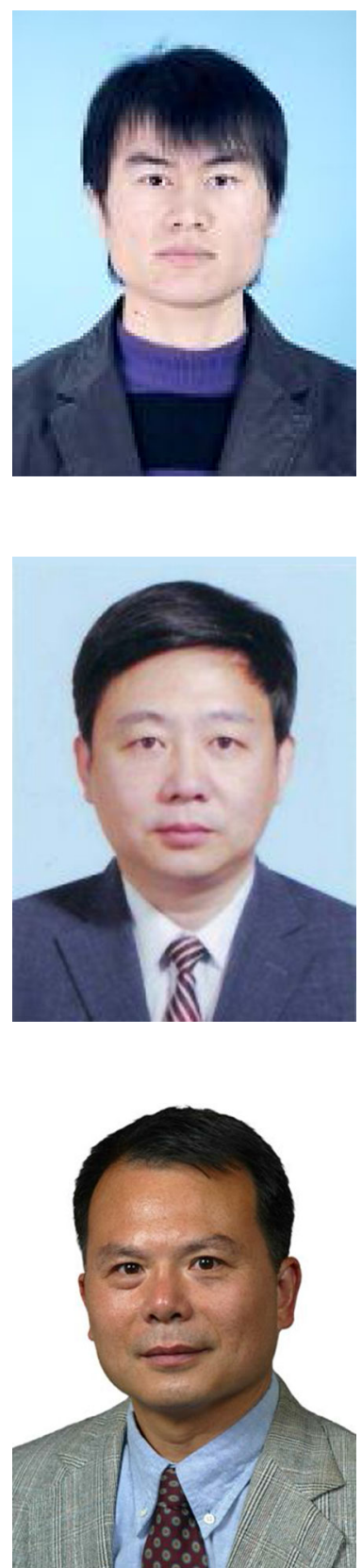

Fuguo Zhao received the BS degree in Logistic Management from Tianjin University of Science and Technology, Tianjin, China in 2011. He is currently a Ph.D. candidate in Management Science and Engineering at University of Science and Technology of China. His research interests include decision science and dual-channel supply chain optimization.

Liang Liang received the Ph.D. degree in System Engineering from Southeast University, Jiangsu, China in 1991. He is currently full professor at School of Management, University of Science and Technology of China. His research interests include decision science and supply chain management.

Zhimin Huang received the Ph.D. degree in Management Science from The University of Texas at Austin in 1991. He is currently full professor at Robert B. Willumstad School of Business, Adelphi University. His research interests include supply chain management and productivity analysis. 